\title{
An intelligent video-monitoring system to detect responsive behaviours associated with Alzheimer's disease and related disorders
}

\author{
Nathalie Tran MSc ${ }^{\mathrm{a}}$ \\ Nolwenn Lapierre $\mathrm{PhD}^{\mathrm{b}, \mathrm{c}}$ \\ Jean Meunier $\mathrm{PhD}^{\mathrm{d}}$ \\ Alain St-Arnaud $\mathrm{MA}^{\mathrm{e}}$ \\ Céline Sit MSc ${ }^{a}$ \\ Anne Bourbonnais $\mathrm{PhD}^{\mathrm{f}, \mathrm{g}}$ \\ Jacqueline Rousseau $\mathrm{PhD}^{\mathrm{a}, \mathrm{b}, *}$
}

\begin{abstract}
${ }^{a}$ School of Rehabilitation, Faculty of Medicine, Université de Montréal, Site Pavillon Parc. C.P. 6128 Centre-ville, Montréal, Québec H3C 3J7, Canada; ${ }^{b}$ Research Center, Institut universitaire de gériatrie de Montréal (Pavillon André-Roch Lecours), 4565 chemin Queen-Mary, Montréal, Québec H3W 1W5, Canada; 'Faculty of Medicine, Université de Montréal, Site Pavillon Parc. C.P. 6128 Centre-ville, Montréal, Québec H3C 3J7, Canada; ${ }^{\text {d}}$ Département d'informatique et de recherche opérationnelle, Université de Montréal, 2900 Boulevard Edouard-Montpetit, Montréal Québec H3T 1J4, Canada; ${ }^{\circ}$ CIUSSS de l'Estde-l'île-de-Montréal 5415, boulevard I'Assomption Montréal, Québec H1T 2M4, Canada; ${ }^{\text {fF} F a c u l t y ~ o f ~}$ Nursing, Université de Montréal, C.P. 6128 Centre-Ville, Montréal, Québec H3C 3J7 Canada; ${ }^{8}$ Research Chair in Nursing Care for Older People and their Families, Research Center, Institut universitaire de gériatrie de Montréal (Pavillon André-Roch Lecours), 4565 chemin Queen-Mary, Montréal, Québec H3W 1W5, Canada; *Corresponding author: jacqueline.rousseau@umontreal.ca
\end{abstract}

N. Tran, N. Lapierre, J. Meunier, A. St-Arnaud, C. Sit, A. Bourbonnais, J. Rousseau. An intelligent video-monitoring system to detect responsive behaviours associated with Alzheimer's disease and related disorders. Gerontechnology 2020;19(1):28-41; https://doi.org/10.4017/gt.2020.19.1.004.00 Background Responsive behaviours affect $90 \%$ of older adults with Alzheimer's disease and related disorders. They have significant consequences including decreased functional independence and quality of life for older adults living in long-term care facilities. An intelligent video-monitoring system (IVS) is being developed to detect responsive behaviours, document their causes and alert health-care professionals to ensure immediate intervention. Purpose To test the IVS's efficacy for responsive behaviour detection. Methods Two occupational therapy students completed a simulation study in an apartment-laboratory under the supervision of experts in gerontology (clinicians, researchers). Four responsive behaviours (aggressiveness, apathy, motor behaviours, vocal behaviours) were realistically replicated across six scenarios, and five were repeated under three different luminosity conditions (total: 16 scenarios). The IVS detects responsive behaviours based on unusual movements or screaming in a specific location. To assess its detection capacity, the scenarios were divided into actions to record true and false positives (TP, FP), and true and false negatives (TN, FN). Sensitivity and specificity were then calculated. The quality of sound, images, and alerts was also analysed. Findings Seventeen TP, two FP, $42 \mathrm{TN}$, and three FN were recorded, generating an overall sensitivity of $85 \%$ and specificity of $95 \%$. Sensitivity and specificity of $100 \%$ were obtained for apathy and aggressiveness scenarios. Motor behaviour scenarios achieved a sensitivity of $75 \%$ and specificity of $84 \%$, and verbal behaviour scenarios obtained a sensitivity of $50 \%$ and specificity of $100 \%$. With this IVS, the quality of the images was satisfactory, but the sound recording was poor. Alerts were received on average 32.4 seconds after detection. Discussion The IVS is an innovative technology that can contribute to responsive behaviour management by immediately detecting such behaviours and helping identify their causes (by recording the previous 30 seconds). These results validate the IVS's potential for responsive behaviour detection before its use is explored in real contexts.

Keywords: Technology, older adults, video-monitoring, responsive behaviour, long-term care, Alzheimer's disease 


\section{INTRODUCTION}

More than $8.5 \%$ of the world's population is currently aged 65 and over (National Institute on Aging, 2016), and by 2050 older people will account for $30 \%$ of the world's population (World Health Organization, 2015). Many health issues tend to manifest themselves with age, including Alzheimer's disease and related disorders (ADRD). ADRD is a degenerative brain disease that can lead to impairments of cognitive function such as memory, language, problem-solving and orientation (Alzheimer's Association, 2017; Alzheimer's Disease International, 2016). ADRD affects 47 million persons in the world (Alzheimer's Disease International, 2016). It can lead to impaired memory, reasoning, orientation, comprehension, learning ability, language and judgment (World Health Organization, 2015).

One of the greatest challenges in taking care of a person with ADRD is the management of responsive behaviours, which occur in $90 \%$ of this population (Alzheimer Society of Canada, 2017; Cerejeira, Lagarto, \& Mukaetova-Ladinska, 2012; Dupuis, Wiersma, \& Loiselle, 2012; Macaulay, 2018). The most common behaviours include apathy, aggressiveness and vocal and motor behaviours (Cerejeira et al., 2012; Zaudig, 2000). Apathy, present in $50 \%$ to $70 \%$ of older adults with ADRD, is a decrease in an initiative to engage in an activity and a loss of interest in social interactions (Ferrero-Arias et al., 2011; Politis et al., 2004). Aggressiveness, which is prevalent in $76.5 \%$ of this population, includes behaviours such as voluntarily hitting others (CohenMansfield, 2008). Motor behaviours are a set of behaviours that deviate from social norms such as wandering and repetitive gestures, and vocal behaviours include shouting and repeating sentences (Cohen-Mansfield, 2008; CohenMansfield \& Libin, 2005). According to Algase et al.'s (1996) theory, older adults demonstrate responsive behaviours as a response to physical or psychological needs that are unmet or compromised, or to communicate these needs to others (Algase et al., 1996). The consequences of responsive behaviours are strongly associated with a significant decline in older adults' functional independence and quality of life (Hersch, 2008; Zaudig, 2000). As a result, prolonged hospital stays and premature institutionalization of older adults with responsive behaviours are common (McCraken, Kagan, \& Parmar, 2009).

Despite the availability of assessment tools to identify the predisposing, precipitating and perpetuating factors of responsive behaviours (e.g., Cohen-Mansfield Agitation Inventory; CohenMansfield, 1997), only a few can be used to document responsive behaviours (Kales, Gitlin, \& Lyketsos, 2015). Non-pharmacological interven- tions (e.g., cognitive stimulation, environmental and psychosocial interventions) are recommended to manage responsive behaviours rather than pharmacological tools, which are not very effective and have major side effects (Hersch, 2008). However, there is a lack of evidence supporting the effectiveness of non-pharmacological interventions and studies show that the effects obtained do not persist over time (Dyer, Harrison, Laver, Whitehead, \& Crotty, 2018; Lorusso \& Bosch, 2018; Möhler, Renom, Renom, \& Meyer, 2018; Scales, Zimmerman, \& Miller, 2018). Medication may be used in combination with non-pharmacological interventions but is not ideal because of the high risk of side effects and medical complications (Hersch, 2008). Physical restraints (e.g., bed rails, belt restraint) are used in $28.2 \%$ of cases of motor behaviours (Bartels et al., 2003; Feteanu, Lopez-Tourres, \& Trivalle, 2005). However, they are strongly associated with negative consequences such as fall risks, injuries and pressure ulcers (Alzheimer's Disease International, 2016; Feteanu et al., 2005).

Recognizing the need to improve the quality of care for older adults with responsive behaviours, several practice guidelines have been published. The Describe, Investigate, Create, Evaluate (DICE) approach ${ }^{\mathrm{TM}}$, a systematic approach developed by US experts, aims to standardize the management of responsive behaviours (Kales et al., 2015). This approach has four steps: (1) describe the responsive behaviour and analyse the occupation during which it occurred; (2) investigate the factors underlying the manifestation of the responsive behaviour; (3) create an interdisciplinary intervention plan; and (4) evaluate the benefits and consequences of the interdisciplinary intervention plan. An intervention approach based on the meaning of behaviour types was also developed and tested for vocal behaviours in long-term care facilities (Bourbonnais, Ducharme, et al., 2018; Bourbonnais, Lalonde, \& Lavallée, 2018). The current systematic approach comprises family and formal caregiver partnerships, which include regular meetings (at least monthly) to identify the meaning of the behaviour (e.g., pain, anxiety) and apply personalized interventions (e.g., gardening, humour) based on this meaning. A pilot study demonstrated the feasibility and acceptability of the approach (Bourbonnais, Ducharme, et al., 2018), which decreased the frequency of behaviours, and increased the well-being of older adults with ADRD. The formal and family caregivers perceived the behaviours as less disruptive and felt more empowered to deal with them.

Monitoring technologies may be useful to facilitate the implementation of approaches or interventions that are often difficult to apply in 
long-term care facilities because of limited resources and the substantial customization they involve. Few technologies have focused on responsive behaviours (Qassem, Tadros, Moore, \& Xhafa, 2014). When such technologies are developed, they mostly focus on wandering behaviours (Neubauer et al., 2018) or are used as an intervention to reduce responsive behaviours, for example using an iPad to reduce agitation (Vahia et al., 2017). For health-care professionals, one mobile application presents guidelines for responsive behaviours (Burns, Jayasinha, \& Brodaty, 2017), but without monitoring or interactive functionalities. No other technologies related to aggressive, apathy, motor or vocal behaviours were found. An intelligent video-monitoring system (IVS) could address these limitations. The aim of this IVS is to detect responsive behaviours continuously (24/7), while still respecting the person's privacy. Consisting of a camera connected to a computer, the IVS automatically sends an alert to the health-care professional's computer or smartphone if a responsive behaviour occurs (e.g., apathy, motor behaviour). An audio signal can be emitted in the room to inform the person that an alert (email) has been sent. The IVS functions in a closed circuit; in the absence of responsive behaviour, no one can access the images. In case of responsive behaviour, images can be accessed in real-time with a password to assess the older adult's situation, and the IVS offers the option of recording the images 30 seconds before the detected event (Rougier, St-Arnaud, Rousseau, \& Meunier, 2011). These images can be automatically processed (blurred) to respect privacy (Lapierre, Meunier, St-Arnaud, \& Rousseau, 2018). Moreover, the IVS uses lowcost equipment and open-access software.

Several factors may affect the effectiveness of camera-based detection systems, including false alarms and system failures (Comité national d'éthique sur le vieillissement, 2015; Niemeijer et al., 2010). Ethical issues surrounding the use of cameras, such as the viewing, transmission and archiving of recordings, could compromise older adults' fundamental rights (Comité national d'éthique sur le vieillissement, 2015; Niemeijer et al., 2010). However, unlike other camera detection systems, the IVS studied here works in a closed circuit: if there is no alert, nobody can access the images, which are analysed in real-time by the computer. Also, no human intervention is required to trigger an alert when an event is detected (Lapierre, Meunier, St-Arnaud, \& Rousseau, 2018). Records are not stored in the cloud; only an assigned person with an identification code and a password may access them. These characteristics specific to the IVS presented here allow user privacy to be respected, unlike detection systems that record continuously (Londei et al., 2009). The IVS's cam- eras are equipped with an infrared illuminator to allow detection even in a poorly lit environment (Rousseau, Meunier, \& Saint-Arnaud, 2011). A study explored health-care professionals' perceptions and needs regarding the potential use of the IVS to detect responsive behaviours in long-term care facilities, and the technology was considered a potentially valuable tool to increase the quality of care and security (Bourbonnais et al., Accepted; Bourbonnais et al., 2017). Furthermore, the study by Lapierre et al. (2016) shows that health-care professionals are also interested in using such technology to detect falls. The overall goal of setting up an IVS in an older adult's living environment is to ensure closer monitoring of the person and provide care immediately upon detection of a responsive behaviour to avoid its exacerbation. The IVS allows the health-care professionals to receive an alert on their smartphone wherever they are so that they can immediately react to the responsive behaviour. Considering the characteristics of responsive behaviours, which must be detected and reported while preserving users' privacy, in addition to health-care professionals' receptivity, the IVS is a promising technology to meet certain existing needs in the health-care system.

The development of the IVS is based on the Model of Competence (Rousseau, 2017; Rousseau, Potvin, Dutil, \& Falta, 2002), which explains the relationship between the person and the environment. Six concepts illustrate the person-environment interaction: (1) person, here the older adult interacting with (2) the environment: human, operationalized as the health-care professional, and nonhuman, operationalized as the long-term care facility and the IVS. These two concepts interact together through (3) an activity, and (4) a role, here care recipient. These interactions can lead to either (5) a competency situation, operationalized here as the activity executed without any responsive behaviour, or (6) a handicap situation, operationalized here as the responsive behaviour. Based on the conceptual framework, the aim of this study was to evaluate the relevance and the potential of the IVS to detect responsive behaviours in older adults living in long-term care facilities.

\section{Methods}

To answer the research question 'Is the intelligent video-monitoring system (IVS) relevant and does it allow users to detect responsive behaviours related to ADRD in older adults living in long-term care facilities?', a simulation study (qualitative and quantitative data) was conducted (Contandriopoulos, Champagne, Potvin, Denis, \& Boyle, 2005).

\section{Participants and procedure}

Scenarios were simulated by three co-authors: two master's students in occupational therapy and one doctoral student in aging sciences, un- 
Table 1. Parameter definition according to iSpyConnect $(C$

Parameter

Definition

Detector type

Trigger range

Gain

Process interval

Mode

Intervals

Recording mode

Buffer

Inactivity record

Min record time

Max record time

Detection zones
Motion detection mode. In this study, '2 frames' mode is used. iSpy(C) compares the last frame to the current one.

Percentage of change in pixels between two consecutive images needed to trigger an alert. For example, an interval of 30 to 65 means that a change of $30 \%$ to $65 \%$ of the pixels between two consecutive images will trigger an alert. A change of greater than $65 \%$ will not trigger an alert to avoid false alerts when the light is switched on or off.

Allows the amplification of movements. The higher the gain, the more sensitive the IVS is to movement.

Reduces the computer's workload. The 'process every 2 frames' parameter has the effect of halving the computer's workload compared to 'process every 1 frame' and increasing the sensitivity to movement by a factor of 2 . Any movement will appear about twice as large with 'process every 2 frames'.

iSpy(C) offers two mode options, either 'motion' or 'no movement'. 'Motion' mode means that iSpy( will trigger an alert if it detects movement while 'no movement' mode means that iSpy( will trigger an alert if it does not detect motion.

Duration for which the IVS will not detect any movement or sound after triggering an alert. For example, an interval of 10 seconds means that there will be no motion or sound detection for 10 seconds after an alert is triggered.

Recording mode after detecting a responsive behaviour. As part of the study, 'record on alert' mode means that the IVS records images or sounds if an alert is triggered.

Recovers the images preceding the triggering of an alert. For example, a 'buffer' of 30 seconds means that the IVS retrieves images from the 30 seconds before an alert is triggered.

Recording time after a motion or sound is detected.

Minimum recording time following an alert.

Maximum recording time following an alert.

Selected areas for which the IVS detects motion (ignoring motion that occurs outside these areas). der the supervision of experts in gerontology and image processing (clinicians, researchers). One or two students (occupational therapy and aging sciences) simulated older adults' activities and falls; the third (occupational therapy) simulated the professional receiving the alert by email on a computer or smartphone. Simulations were performed in an apartment-laboratory designed to reproduce the bedroom and living room of an older adult living in a long-term care facility. One complete IVS was installed per room (camera and computer). A calibration phase was needed to configure the IVS depending on environmental factors (e.g., the room's size and lighting) and the participant simulating the responsive behaviour (e.g., height, weight). The calibration involved trial and error to determine the optimum thresholds for each parameter (presented in Table 1).

After the calibration phase, four responsive behaviours (aggressiveness, apathy, motor behaviours, and vocal behaviours) were simulated across six scenarios (Table 2). Each scenario, with the exception of vocal behaviours, was reproduced at three different luminosity conditions: (1) artificial lighting: Lights on, curtains and doors closed; (2) night vision: lights off, curtains and doors closed; (3) daylight: lights off, curtains and doors open. Thus, a total of 16 simulations were executed.

\section{Material and equipment}

The IVS consisted of a camera with infrared illuminator (1 per room) and a wide-angle lens, a laptop and telescopic rods to support the cameras. Because the camera did not have a built-in microphone, the laptop's microphone was used for sound detection. Each computer was equipped with iSpyConnect $@$ (open-source camera security software) and Mathematica $($ ) (software to complement iSpy()). For alert transmission, a Wi-Fi connection was used. Following the detection of a responsive behaviour, different types of alerts, including an audio signal and email were triggered. The email sent included a realtime image of the participant (except for the vocal behaviour detection). To protect privacy and security, real-time images can only be accessed with a password. The date and time of the detection of responsive behaviour and the words 'confidential image' were indicated in the alert. The image attached to the email had a resolution of 320 × 240 pixels. For the vocal behaviours scenario, an email was sent with the subject 'scream detected'. The IVS was programmed to record 30 seconds of sounds (only for vocal behaviours) or images before the responsive behaviour to 
Table 2. Description of scenarios

Scenario $\begin{gathered}\text { Responsive behaviours } \\ \text { simulated }\end{gathered}$

$1 \quad$ Motor behaviours

Motor behaviours

3

Apathy

4

Apathy

5

Aggressiveness

6
Description

Operations
The older adult is sitting in the bedroom with her hands on a table in front of her. She eats, drinks, scratches her head and knocks her fist on the table several times. Upon the detection of signs of motor behaviours, an alert is sent to the health-care professional's smart phone.

The older adult is standing next to the bed in the bedroom. She lifts the blanket and goes to bed. She turns slowly and then moves dangerously in her bed. Upon the detection of signs of motor behaviours, an alert is sent to the healthcare professional's smart phone.

The older adult is sitting in the bedroom. She yawns and scratches her head. She remains motionless for 10 minutes. Upon the detection of signs of apathy, an alert is sent to the health-care professional's smart phone.

The older adult is sitting in the bedroom and remains motionless for 10 minutes while a resident approaches her for a brief conversation. Upon the detection of signs of apathy, an alert is sent to the health-care professional's smart phone.

Two older adults are sitting at opposite ends of the living room. One gets up, walks over to the other one and hits her. Upon the detection of signs of aggressiveness, an alert is sent to the health-care professional's smart phone.

The older adult is sitting in the room and has a brief conversation with a healthcare professional, who slams the door as she leaves the room. The older adult shouts for at least 2 seconds. Upon the detection of the signs of vocal behaviours, an alert is sent to the healthcare professional's smart phone.
To eat a meal, consume a drink, scratch the head, and knock on the table.

To lift the blanket, get into bed, lie down under the blanket and turn around ir the bed.

To yawn and scratch the head.

To walk and have a conversation.

To walk and show signs of aggressiveness.

To have a conversation and slam the door. document its triggering context.

Two IVS setups were installed: one in the bedroom and the other in the living room. The distance separating the IVS from the older adult was $3.0 \mathrm{~m}$ and $0.8 \mathrm{~m}$, respectively. Each IVS was located $2.5 \mathrm{~m}$ above the ground in the upper corner of the room to maximize the viewing angle and above the window, so that exterior luminosity changes would not interfere with the system. Its installation on a ceiling corner at an optimum distance from the older adult was essential to reduce the effect of perspective projection (i.e., motion far from the camera appears smaller than motion close by). Conversely, if the IVS was installed lower down, any movement near the IVS would have created a greater change in the number of pixels than the same movement at a greater distance. This could have induced more false positives. Installation of the IVS on the wall above windows was also important to minimize the presence of shadows that could be interpreted as movement. A total of 12 iSpy@ parameters were used. The definition of each one, with some explanations, is shown in Table 3. These parameters vary depending on several factors, 
Table 3. Parameters obtained for each scenario

\begin{tabular}{|c|c|c|c|c|c|c|}
\hline & $\begin{array}{l}\text { Scenario 1: } \\
\text { Motor } \\
\text { behaviours }\end{array}$ & $\begin{array}{c}\text { Scenario 2: } \\
\text { Motor } \\
\text { behaviours }\end{array}$ & $\begin{array}{l}\text { Scenario 3: } \\
\text { Apathy }\end{array}$ & $\begin{array}{l}\text { Scenario 4: } \\
\text { Apathy }\end{array}$ & $\begin{array}{c}\text { Scenario 5: } \\
\text { Aggressiveness }\end{array}$ & $\begin{array}{c}\text { Scenario 6: } \\
\text { Vocal behaviours }\end{array}$ \\
\hline $\begin{array}{l}\text { Detection } \\
\text { type }\end{array}$ & 2 frames & 2 frames & 2 frames & 2 frames & 2 frames & $\mathrm{n} / \mathrm{a}$ \\
\hline $\begin{array}{l}\text { Trigger } \\
\text { range }\end{array}$ & 30 to 65 & 30 to 65 & 30 to 65 & 30 to 65 & 30 to 65 & 40 to 100 \\
\hline Gain & 10 & 10 & 10 & 10 & 10 & 3 \\
\hline $\begin{array}{l}\text { Process } \\
\text { interval }\end{array}$ & 1 frame & 1 frame & 1 frame & 1 frame & 2 frames & $\mathrm{n} / \mathrm{a}$ \\
\hline $\begin{array}{l}\text { Recording } \\
\text { mode }\end{array}$ & $\begin{array}{l}\text { Record on } \\
\text { alert }\end{array}$ & $\begin{array}{l}\text { Record on } \\
\text { alert }\end{array}$ & $\begin{array}{l}\text { Record on } \\
\text { alert }\end{array}$ & $\begin{array}{l}\text { Record on } \\
\text { alert }\end{array}$ & $\begin{array}{l}\text { Record on } \\
\text { alert }\end{array}$ & $\begin{array}{c}\text { Record on alert } \\
\text { when sound is detected } \\
\text { for } 2 \mathrm{~s}\end{array}$ \\
\hline Mode & $\begin{array}{l}\text { Movement } \\
\quad(\mathrm{mvt})\end{array}$ & Mvt & $\begin{array}{l}\text { No mvt for } \\
600.0 \mathrm{~s}\end{array}$ & $\begin{array}{l}\text { No mvt for } \\
600.0 \mathrm{~s}\end{array}$ & Mvt & $\mathrm{n} / \mathrm{a}$ \\
\hline Intervals & $10.0 \mathrm{~s}$ & $10.0 \mathrm{~s}$ & $10.0 \mathrm{~s}$ & $10.0 \mathrm{~s}$ & $10.0 \mathrm{~s}$ & $60.0 \mathrm{~s}$ \\
\hline Buffer & $30.0 \mathrm{~s}$ & $30.0 \mathrm{~s}$ & $30.0 \mathrm{~s}$ & $30.0 \mathrm{~s}$ & $30.0 \mathrm{~s}$ & $0 \mathrm{~s}$ \\
\hline $\begin{array}{l}\text { Inactivity } \\
\text { record }\end{array}$ & $10.0 \mathrm{~s}$ & $10.0 \mathrm{~s}$ & $10.0 \mathrm{~s}$ & $10.0 \mathrm{~s}$ & $10.0 \mathrm{~s}$ & $10.0 \mathrm{~s}$ \\
\hline $\begin{array}{l}\text { Min } \\
\text { record } \\
\text { time }\end{array}$ & $0 \mathrm{~s}$ & $0 \mathrm{~s}$ & $0 \mathrm{~s}$ & $0 \mathrm{~s}$ & $0 \mathrm{~s}$ & $0 \mathrm{~s}$ \\
\hline $\begin{array}{l}\text { Max } \\
\text { record } \\
\text { time }\end{array}$ & $60.0 \mathrm{~s}$ & $60.0 \mathrm{~s}$ & $60.0 \mathrm{~s}$ & $60.0 \mathrm{~s}$ & $60.0 \mathrm{~s}$ & $60.0 \mathrm{~s}$ \\
\hline $\begin{array}{l}\text { Detection } \\
\text { zones }\end{array}$ & 5 & 5 & 1 & 1 & 1 & $\mathrm{n} / \mathrm{a}$ \\
\hline
\end{tabular}

including the participants' characteristics (e.g., height, weight, the pattern of normal activities) and the characteristics of the non-human environment (e.g., light intensity, presence of shadows, location of furniture). The 'process interval' parameter was used to increase the sensitivity of the movement detection by a factor of 2 as the percentage of change in pixels between two consecutive images associated with the simulated behaviours (e.g., one older adult hitting another one) was sometimes not sufficient to trigger an alert. Thus, when the IVS was configured with the 'process interval' parameter at 2, the movement was measured as being about twice as large as if the 'process interval' had been at 1. To evaluate the IVS's capacity to detect apathy, the 'no movement for 600 seconds' mode was configured, meaning that an absence of motion within the 'detection zone' for 600 seconds would trigger an alert.

\section{Data collection}

For each scenario simulated, one or two participants (depending on the scenario) simulated the older adult(s) and another received the alert on the laptop or smartphone and completed an Excel spreadsheet to collect qualitative and quanti- tative data about its installation, calibration, and functioning. The spreadsheet was based on the content of the Home Assessment of Person-Environment Interaction (HoPE) (Rousseau, 2016; Rousseau, Potvin, Dutil, \& Falta, 2013), which is based on the Model of Competence (Rousseau, 2017; Rousseau et al., 2002). Data related to the scenario were collected (e.g., characteristics of the physical environment, IVS location). The intensity of brightness and the presence of shadowy areas within the apartment-laboratory were noted. For the installation of the IVS, data were collected regarding the number of persons, time and equipment required to install the entire IVS. For the ease of configuration of the IVS, data were collected regarding the number of parameters to be configured and the need to adjust them according to the simulation scenarios. Alerts triggered by the IVS and their causes were documented in the grid. Time elapsed between detection by the IVS and the receipt of the alert (by email) was manually noted for each simulation scenario. Images were viewed and sound recordings listened to, to determine if their quality was satisfactory and allowed the recipient to respond upon receiving the alert. 


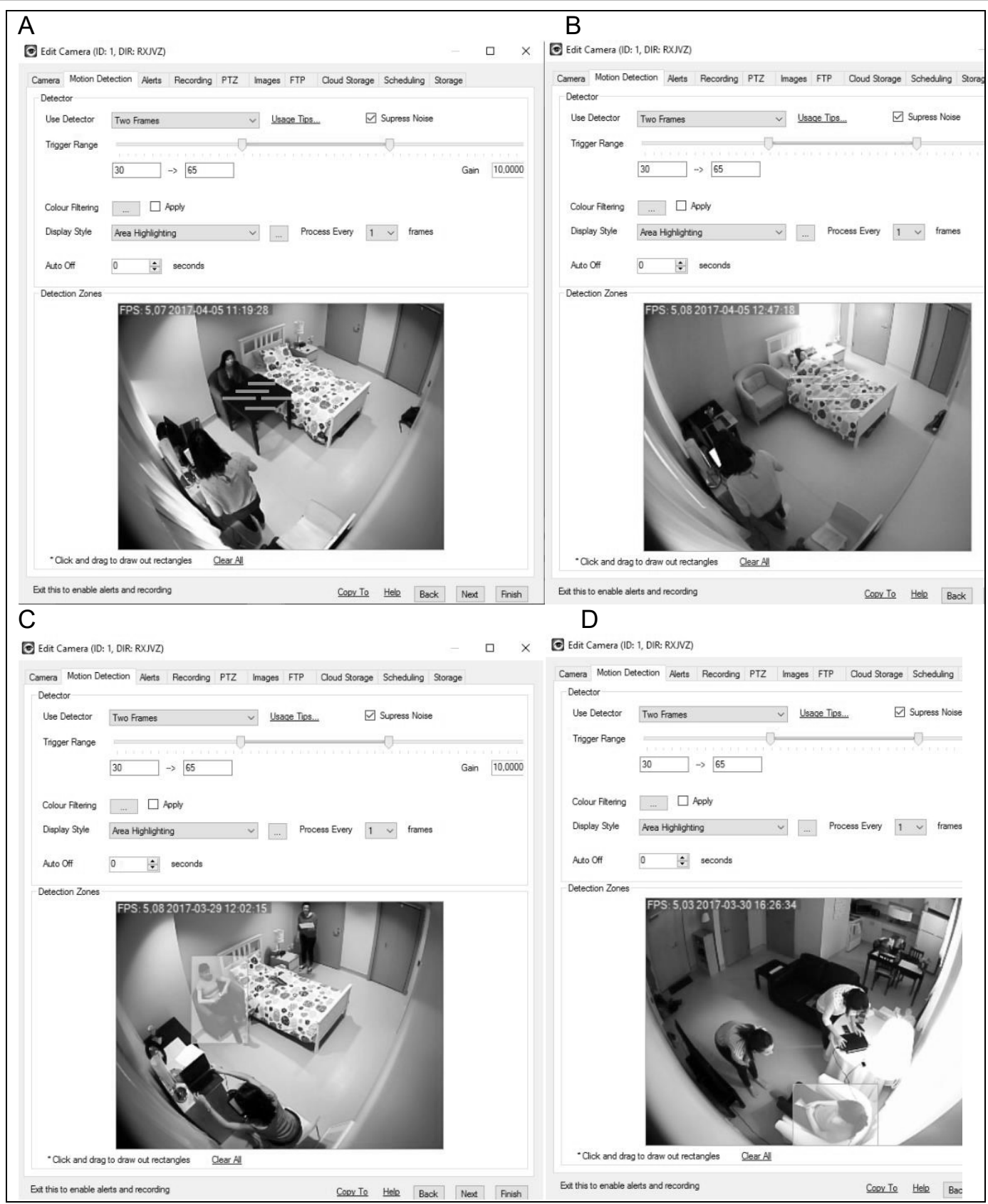

Figure 1. Examples of parameters. (a) scenario 1, (b) scenario 2, (c) scenario 3, and (d) scenario 4 (left to right and top to bottom: $a, b, c, d$ ).

\section{Data analysis}

Qualitative and quantitative data on the installation, calibration, and ease of use of the system in the apartment-laboratory were descriptively analysed (e.g. installation for each room was timed and described). The mean time for installation and calibration was calculated. The quality of the sound recording was considered satisfactory if it was a realistic representation of the older adult's voice and did not have any abnormal volume variation. The image quality was considered satisfactory if there was no significant distortion of the image caused by the wide-angle of the camera and it was clear enough to assess the older adult's situation after the detection. Regarding the IVS's effectiveness at detecting responsive behaviours, in order to calculate true positives (TP), false negatives (FN), false positives (FP) and 
true negatives (TN), scenarios were broken down into operations (actions) as defined by the HoPE tool, and some other actions were added since they were likely to trigger an alert. They were calculated as follows: TP $=$ number of responsive behaviours correctly detected and for which an email was received; $\mathrm{FN}=$ number of responsive behaviours not detected or for which an email was not received; FP = number of 'normal' operations identified as responsive behaviours; TN = number of 'normal' operations not identified as responsive behaviours. For each simulated scenario, the numbers of TP, TN, FP, and FN were counted to determine the IVS's specificity (Sp) and sensitivity (Se). Se of the IVS was calculated as follows: $\mathrm{Se}=\mathrm{TP} /(\mathrm{TP}+\mathrm{FN})$. The $\mathrm{sp}$ of the IVS was calculated as $\mathrm{Sp}=\mathrm{TN} /(\mathrm{TN}+\mathrm{FP})$.

\section{Results}

The results are presented under three headings: (1) Installation and configuration of the IVS, (2) Sensitivity and specificity of the IVS, and (3) Alerts, image and sound quality.

\section{Installation and configuration of the IVS}

On average, installation took 5 minutes and was considered easy due to the small number of components to install (camera, computer, and telescopic rod). The configuration of the IVS during the calibration phase was laborious because of the numerous parameters to adjust. During the initial calibration phase, the size and number of 'detection zones' and the 'trigger range' parameter were particularly difficult to set and required several trials. The feasibility of the IVS to detect responsive behaviours in older adults living in long-term care facilities was assessed, among other ways, by determining if it was possible to obtain common technical parameters for all scenarios. For this purpose, parameters were compared. The parameters obtained for each scenario are reported in Table 3.

Parameters for the motor behaviour scenarios (scenarios 1 and 2) were identical, and the same number of detection zones (5 zones per scenario) was needed even though the cameras were not located in the same place in the two scenarios (Figures $1 a$ and $1 b$ ).

For the apathy scenarios (scenarios 3 and 4), all the parameters were identical, including the 'detection zones' even though the two scenarios did not occur in the same room (Figure 1c). Both 'detection zones' corresponded to a rectangle completely covering the older adult. Comparing the motor behaviour scenarios with the apathy scenarios, only the 'mode' and 'detection zones' parameters differed: for scenarios 1 and 2, the mode was set to 'movement' with five 'detection zones', while scenarios 3 and 4 used the 'no movement for 600 seconds' mode and one detection zone. Only one detection zone was necessary for the apathy scenario as the goal was to detect the absence of movement (e.g., apathy) where the participant was sitting. For all scenarios simulated in the bedroom (scenarios 1, 2, and 3), all parameters were identical except for the 'detection zones' and 'mode'. For scenarios simulated in the living room (scenarios 4 and 5), only the 'mode' and 'process interval' parameters were different. Scenario 5 was the only one to have the 'process interval' parameter set at 2 frames (Figure 1d). The parameters for the vocal behaviour scenario (scenario 6) are reported in Table 3; they focused on detecting specific sounds, whereas the IVS was set to analyse images for the other scenarios.

\section{Sensitivity and specificity of the IVS}

Table 4 shows the number of TP, FP, TN, and FN obtained per scenario. A total of 17 TP, 2 FP, 42 $\mathrm{TN}$, and $3 \mathrm{FN}$ were recorded, leading to an overall Se of $85 \%$ and Sp of $95 \%$. Four out of six scenarios achieved Se and Sp of 100\% (apathy and aggressiveness scenarios). Vocal behaviour scenarios obtained Se of $50 \%$ and Sp of $100 \%$; responsive behaviours were detected in both scenarios 4 and 5 (apathy and aggressiveness), and the audio signal was emitted but no email was sent. The motor behaviour scenarios had Se of $75 \%$ and Sp of $84 \%$; no email was sent despite the detection of responsive behaviours when the lighting was artificial. Moreover, two alerts occurred in the absence of responsive behaviour when the lighting was natural.

\section{Alerts, image, and sound quality}

The time elapsed between the detection of the responsive behaviour and the receipt of the alert varied between 20.3 seconds and 56.4 seconds among the 16 simulations. On three occasions, no alert was received despite the detection of responsive behaviour (scenarios 2 and 6). By excluding these three simulations, the mean time between the detection of a responsive behaviour and the receipt of the alert was 32.4 seconds for the 13 simulations. On average, the scenario for which the alert was received the fastest was scenario 5 (mean of 24.1 seconds for three simulations with different luminosity for each), whereas the scenario for which the alert was received the slowest was scenario 1 (mean of 51.9 seconds for three simulations). The image covered the entire room in which the IVS was installed and did not show significant distortion. The image was clear enough to recognize the furniture and the room. The facial features of participants (e.g., eyes, mouth and nose) were difficult to distinguish, but it was possible to recognize them by their silhouette and clothing. The images captured in artificial light had an orange hue (Figure 
Table 4. Sensitivity and specificity for each scenario

\begin{tabular}{lcccccc}
\hline & TP & FP & TN & FN & Se & Sp \\
\hline Scenario 1 & 3 & 0 & 9 & 0 & $100 \%$ & $100 \%$ \\
Scenario 2 & 3 & 2 & 11 & 1 & $75 \%$ & $84 \%$ \\
Scenario 3 & 3 & 0 & 6 & 0 & $100 \%$ & $100 \%$ \\
Scenario 4 & 3 & 0 & 6 & 0 & $100 \%$ & $100 \%$ \\
Scenario 5 & 3 & 0 & 6 & 0 & $100 \%$ & $100 \%$ \\
Scenario 6 & 2 & 0 & 4 & 2 & $50 \%$ & $100 \%$ \\
Total & 17 & 2 & 42 & 3 & $85 \%$ & $95 \%$ \\
\hline
\end{tabular}

2a) whereas those made in natural light had a purple hue (Figure $2 b$ and $2 c$ ) due to the infrared illuminator active at this luminosity. Regarding the recording of vocal behaviours, it was of poor quality: the conversation was jerky. Moreover, the words were not audible, which made the recording difficult to understand. However, it was possible to distinguish who was talking.

\section{Discussion}

The aim of this study was to evaluate the relevance and the potential of the IVS to detect responsive behaviours in older adults living in long-term care facilities. The effectiveness demonstrated by the IVS to detect responsive behaviours in an apartment-laboratory and its ease of use support its potential for use with older adults in long-term care. Five aspects of the results are discussed: (1) Installation and configuration of the IVS, (2) Effectiveness of the IVS, (3) Alerts, image and sound analysis, (4) Ethical considerations, and (5) Strengths and limitations.

\section{Installation and configuration of the IVS}

The IVS was fast and easy to install, as it has only three components to assemble. The configura- tion of the IVS was complex and required the collaboration of a professional with computer know-how. However, the use of the IVS, once the calibration is completed, was simple and intuitive. According to Holden et al. (2012), the complexity of technology does not influence health-care professionals' intent to use it as long as they perceive the benefits it could provide for their practice (Holden \& Karsh, 2010). Moreover, studies exploring health-care professionals' perception of the use of the IVS indicate that they favour its implementation; its benefits outweigh the potential difficulties it may present (Bourbonnais et al., 2017; Lapierre et al., 2016). The telescopic rod and the computer were accessible and could represent a safety risk for people entering the room (e.g., risks of falling due to the presence of the computer power cord). The telescopic rod and the computer could also be removed by older adults with ADRD, who tend not to collaborate. Miniaturization of the IVS could optimize its application in long-term care facilities, as the camera could be attached to the ceiling and a wireless camera could be used. However, the IVS already appears to be relevant for health-care professionals because no intervention is required from the older adult to send an alert.

\section{Effectiveness of the IVS}

Two alerts were triggered when the older adult turned over in bed in daylight. These two FPs can be explained by the high intensity of natural light, which created shadows on the bed when there were only slight movements of the blanket. The changes in pixel brightness associated with the appearance of the shadows were sufficient to trigger an alert. This problem did not appear with artificial lighting or at night since no (or negligible)

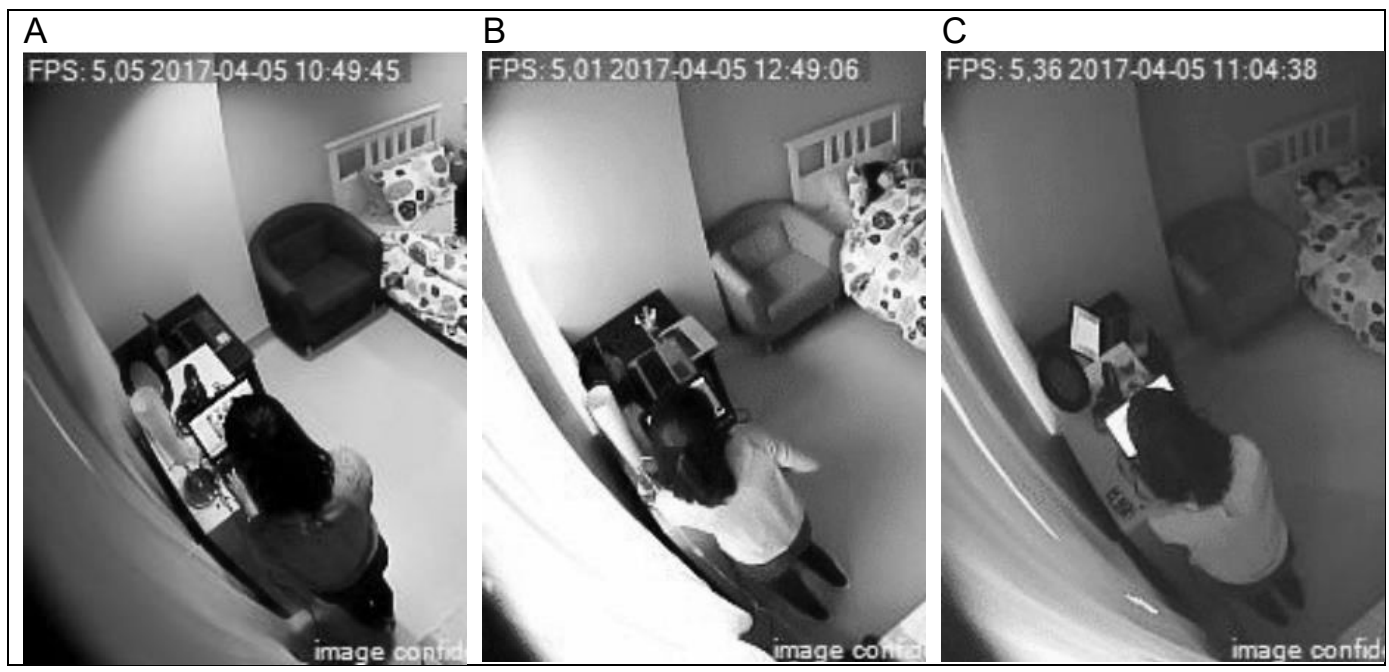

Figure 2. Examples of images sent with the alert. (a) Screenshot of the bedroom with artificial light. (b) Screenshot of the bedroom with daytime light. (c) Screenshot of the bedroom with night-time light (infrared illumination) (left to right: $a, b, c$ ). 
shadow was created. Reducing the size of 'detection zones' and raising the first threshold of the 'trigger range' parameter are solutions that could be combined to lessen the IVS's sensitivity to shadows. Specifically, for situations involving motor behaviours in bed, using a coloured blanket (e.g., blue or dark grey) could reduce shadows. A drawback of the alert mode selected in the project is that receiving emails requires a permanent connection to a Wi-Fi network. Thus, a slowdown or interruption of the Wi-Fi connection could cause the non-receipt of emails despite the detection of responsive behaviour. In scenarios 2 and 6 (motor behaviours and vocal behaviours), the audio signal was emitted but the email was not sent, which explains the Se of $75 \%$ and $50 \%$ obtained. To overcome this problem, it would be possible to select an alternative alert mode via iSpy@ $\bigodot_{\text {, for }}$ example, an audible alert mentioning 'responsive behaviour detected' in the nursing station or a text message in addition to the audio signal. A limitation of some camera detection systems is that they are designed for an environment where only one person lives, which is inappropriate in an environment such as long-term care facilities. The IVS solved this with the 'detection zones' parameter, which can monitor important areas of activity and ignore any movement outside these areas. A long-term care facility is known to be an environment where many interactions among older adults, caregivers, and family members occur. This situation was tested in scenarios 4 and 5 in order to represent the reality of a long-term care facility as participants entered the detection zone but did not trigger a false alert.

\section{Alerts, image and sound analysis}

Despite their low resolution, the quality of the images was considered satisfactory. In all luminosity levels tested, the older adult and the room were easily recognizable. In addition, low-resolution images require little storage space, which minimizes the delay between the detection of responsive behaviour and the receipt of the email. However, when asked about the integration of IVS in practice in home care, participants reported the need to view clear images and be able to enlarge them (Lapierre et al., 2016). iSpy@ offers the possibility of increasing the resolution of the image, allowing a better assessment of the older adult's status. Moreover, the health-care professional could enlarge the image obtained by email on a smartphone or computer. In addition to fitting into a non-pharmacological approach, the 30-second recording before the alert is a feature of the IVS that could help to implement a client-centred intervention adapted to the needs of older adults living in long-term care facilities, such as the DICE approach $^{\mathrm{TM}}$ (Cerejeira et al., 2012; Fraker, Kales, Blazek, Kavanagh, \& Gitlin, 2014; McCraken et al., 2009). This could result in a better assessment of the person-environment interaction and is coherent with the 'Describe' and 'Investigate' steps of the DICE approach and the approach developed by Bourbonnais and others (Bourbonnais, Ducharme, et al., 2018; Kales et al., 2015; Rousseau et al., 2002). An exhaustive description of the responsive behaviour and its context could provide information needed for interventions adapted to the individual's needs and would contribute to the application of non-pharmacological approaches as first-line treatment (Lorusso \& Bosch, 2018; Möller, Kristensson, Midlöv, Ekdahl, \& Jakobsson, 2014; Scales et al., 2018). The 30-second recording before the event could, therefore, be particularly useful to understand the behaviours of older people living in long-term care facilities. In this regard, it is possible to say that the IVS is a component of the non-human environment favouring a person's competency situation (Rousseau, 2017; Rousseau et al., 2002). On the other hand, this function may be less relevant for apathy since the alert triggered by a lack of movement is sufficient to infer that an older adult is showing apathy. The IVS's ability to activate sound recording 30 seconds before a vocal behaviour is an advantage. Any sound of lower intensity and shorter duration than a vocal behaviour (e.g., door closing, conversation) is automatically ignored. However, the computer microphone's sound recording was of poor quality and was considered unsatisfactory given the jerkiness of the conversation. This could be explained by a non-optimal location of the microphone, which was placed $3.0 \mathrm{~m}$ from the older adult during the simulation of vocal behaviours. In future studies, it would be relevant to explore the use of an external high-quality microphone to avoid such problems. It could be centred on the ceiling of the room in order to detect sound regardless of the older adult's position relative to the microphone.

The brief delay of 32.4 seconds between the detection of a responsive behaviour and the receipt of the email was a strength of the IVS, as it would allow health-care professionals to intervene quickly to prevent the exacerbation of the behaviour. Early intervention is paramount given the consequences responsive behaviours entail. Vocal and motor behaviours and aggressiveness, in addition to being indications of ill-being and having potential consequences for others, are related to a greater use of restraint and medication, a decline in functional independence, and an increased risk of falls and social isolation (Bourbonnais, Lalonde, et al., 2018; Scales et al., 2018). Moreover, the study by Treusch et al., (2015) indicates that apathy is often overlooked by health-care professionals compared with other responsive behaviours because it causes less professional burden. Yet the consequences of a long period of inactivity are severe: a period of immobility longer than two hours exposes an 
older adult to a significant risk of pressure ulcers (Bourque et al., 2012). Thus, detecting apathy is particularly important in a long-term care facility to prevent pressure ulcers.

\section{Ethical considerations}

The Comité national d'éthique sur le vieillissement (2015) has raised concerns about the privacy, confidentiality, and dignity of older adults in the context of the use of camera-based technologies, especially those requiring continuous recording. However, the IVS studied here has several functions that can address these issues. First, it operates in a closed circuit; detection of the responsive behaviour does not involve a person watching the older adult or continuous recording. The recording starts only when a responsive behaviour is detected and ends after the number of seconds determined in advance by the health-care professional. Any image or sound that does not trigger an alert is automatically deleted. Furthermore, recorded data are stored locally and secured by a password; they are not transmitted to the cloud. Future IVS studies should consider the possibility of deleting images and sounds stored on the local server after a specified period of time. The Comité national d'éthique sur le vieillissement (2015) also raised concerns that older adults' privacy and dignity could be compromised by the delivery of health care in the presence of a camera. Although this feature was not studied in our study, it is possible to set a time schedule to automatically disable the IVS (e.g., during the morning hygiene routine), and automatically reactivate it (e.g., after dinner, if the older adult only demonstrates responsive behaviours during the night). In future studies, blurring subjects' faces may be considered to ensure their privacy and confidentiality; we used this strategy in another study (Lapierre, Meunier, St-Arnaud, Filiatrault, et al., 2018). Finally, it is essential to set local normative and legal measures to ensure respect for the fundamental rights of older adults. This is also consistent with the opinion of the health-care professionals who participated in the study by Lapierre et al. (2016): they stated that a protocol regarding the use of IVS is required to avoid ethical problems.

\section{Strengths and limitations}

The IVS has five key strengths and three limitations. The drawbacks are the unsatisfactory sound quality, the interpretation of some shadow motions as movement, generating false alarms, and the receipt of the alert depending on $\mathrm{Wi}-\mathrm{Fi}$ connectivity. This latter aspect is a drawback for most gerontechnologies and needs to be addressed to ensure their accessibility for older adults. Nevertheless, solutions have been identified to overcome these shortcomings. Among the IVS's advantages, the most important are its closed-circuit function, which contributes to the respect of older adults' privacy and confidentiality, its ability to detect and adjust to the luminosity of the room, the speed of the alert receipt (less than 1 minute) and the 30-second recording helping to document the context of the responsive behaviour. The results also show that the IVS is particularly suitable to detect signs of apathy. To facilitate the implementation of the IVS, a cost-effective option is essential. The IVS has a fixed cost (camera, computer, and telescopic rod to support the camera) of about $\$ 500$ in addition to an annual fee of $\$ 1,350$ for the Mathematica $\odot$ license. The latter is not essential since the Mathematica $\odot$ codes could easily be converted into another (free) language. If that were done, the cost of the ISV would typically be reduced to $\$ 500$, making this technology more affordable. The cost would also decrease with a permanent setup, as it would not require the use of a telescoping rod.

No previous study had focused on the effects of monitoring technology regarding responsive behaviours, except in the case of wandering. Our study focused on the monitoring of aggressive, apathetic, motor or vocal behaviours. Biases, which may occur while simulating responsive behaviours, could represent another limitation (e.g., simulations were done by co-authors, who may have behaved in such a way as to improve the results). However, the scenarios were inspired by the content of the HoPE tool (Rousseau et al., 2013), which is based on the Model of Competence (Rousseau, 2017; Rousseau et al., 2002) and performed by students in occupational therapy (who are trained to intervene with older adults manifesting responsive behaviours). An occupational therapist and a neuropsychologist with expertise in gerontology supervised this simulation study, which contributed to simulating realistic scenarios. In addition, the use of an observation spreadsheet based on the HoPE and related to the Model of Competence contributed to the rigour of the study (Rousseau et al., 2013). The analysis of the IVS's specificity and sensitivity was based on breaking down the scenarios into operations (actions). However, the results presented in Table 4 must be interpreted with caution due the small sample size and the frequent occurrence of zeroes; consequently, they do not allow for sophisticated statistical analysis. At this stage of the IVS's development, the focus was on its relevance, so only a small number of scenarios were tested in this preliminary simulation study. Finally, the apartment-laboratory was designed to reproduce the non-human environment in long-term care facilities, which made this study more realistic. A limitation of the study is that the camera was physically disconnected from the laptop during the simulation of vocal behaviours. Although the detection of vocal be- 
haviours does not require the use of cameras, it would have been relevant not to interfere with the system in order to assess the IVS's ability to detect sound and motion simultaneously.

\section{Conclusion}

This study showed that the IVS is relevant for detecting responsive behaviours in older adults living in long-term care facilities. Despite some drawbacks, the results show that the IVS is a promising tool to monitor responsive behaviours and facilitate their management because of its potential to detect them, document their causes and alert health-care professionals when they occur. Four conclusions can be highlighted from this study: (1) Many parameters can be customized according to the particular needs of both the older adult and the health-care professional (e.g., 'detection zones'), which makes the IVS flexible. (2) The IVS's efficacy is optimized under the following conditions: (a) it should be installed in a corner of the room's ceiling; (b) the lighting of the room must create as little shadow as possible in the camera's field of view; and (c) the $\mathrm{Wi}-\mathrm{Fi}$ connection must be adequate to allow the receipt of the alert or an alternative should be considered (SMS or audio signal). (3) The IVS is particularly suitable for detecting apathy. (4) Local ethical procedures should be implemented regarding the use of the IVS to ensure respect for the fundamental rights of older adults with a loss of autonomy.

Future studies should focus on exploring the other options mentioned (e.g., sending SMS alerts, use of an external microphone) to address the issues encountered in this study (e.g., non-receipt of the alert, low-quality sound recording). It is also important to study the IVS's ability to detect responsive behaviour in a group of many older people. The results of such studies would complement this study and take an additional step towards the possible implementation of the IVS in long-term care facilities.

\section{References}

Algase, D. L., Beck, C., Kolanowski, A., Whall, A., Berent, S., Richards, K., \& Beattie, E. (1996). Needdriven dementia-compromised behavior: An alternative view of disruptive behavior. American Journal of Alzheimer's Disease, 11(6), 10-19. https://doi.org/10.1177/153331759601100603

Alzheimer Society of Canada. (2017). Person centred language guidelines (p. 8). Retrieved from http:// alzheimer.ca/sites/default/files/2017-11/Person_ Centred_Language_Guidelines-e.pdf

Alzheimer's Association. (2017). 2017 Alzheimer's disease facts and figures (pp. 325-373). Retrieved from http://www.sciencedirect.com/science/article/pii/S1552526017300511

Alzheimer's disease international. (2016). World Alzheimer Report 2016 - Improving healthcare for people living with dementia: Coverage, quality and costs now and in the future (p. 140).

Bartels, S. J., Horn, S. D., Smout, R. J., Dums, A. R., Flaherty, E., Jones, J. K., Voss, A. C. (2003). Agitation and depression in frail nursing home elderly patients with dementia: treatment characteristics and service use. The American Journal of Geriatric Psychiatry: Official Journal of the American Association for Geriatric Psychiatry, 11(2), 231-238.

Bourbonnais, A., Ducharme, F., Landreville, P., Michaud, C., Gauthier, M.-A., \& Lavallée, M.H. (2018). An Action Research to Optimize the Well-Being of Older People in Nursing Homes: Challenges and Strategies for Implementing a Complex Intervention. Journal of Applied Gerontology, 0733464818762068. https://doi. org/10.1177/0733464818762068

Bourbonnais, A., Lalonde, M.-H., \& Lavallée, M.-H. (2018). Decibels Intervention: Intervention approach based on the meanings of vocal behaviours of older people living with a neurocognitive disorder- Manual for family and formal caregivers (2ème). Montréal: Research Chair in Nursing Care for Older People and their Families.

Bourbonnais, A., Rousseau, J., Lalonde, M-H., Meunier, J., Lapierre, N. et Gagnon, M-P. (Accepted). Perceptions and needs regarding technologies in nursing homes: An exploratory study. Health Informatics Journal.

Bourbonnais, A., Rousseau, J., Meunier, J., Gagnon, M., Lalonde, M., Lapierre, N., de, U. (2017). Behavioral symptoms and falls in long-term care facilities: Perceptions of Gerontechnology. Innovation in Aging, 1(suppl_1), 287-287. https://doi.org/10.1093/geroni/ igx004.1061

Bourque, M., Québec (Province), Ministère de la santé et des services sociaux, Institut universitaire de gériatrie de Montréal, \& Centre de santé et de services sociaux-Institut universitaire de gériatrie de Sherbrooke. (2012). Déclin fonctionnel dans les AVQ. Retrieved from http://collections.banq.qc.ca/ ark:/52327/2107974

Burns, K., Jayasinha, R., \& Brodaty, H. (2017). Evaluation of an Electronic App Developed to Assist Clinicians in the Management of Behavioral and Psychological Symptoms of Dementia (BPSD). International Journal of Human-Computer Interaction, 33(11), 902-910. https://doi.org/10.1080/104 47318.2017.1296075

Cerejeira, J., Lagarto, L., \& Mukaetova-Ladinska, E. B. (2012). Behavioral and Psychological Symptoms of Dementia. Frontiers in Neurology, 3. https://doi. org/10.3389/fneur.2012.00073

Cohen-Mansfield, J. (1997). Conceptualization of Agitation: Results Based on the Cohen-Mansfield Agitation Inventory and the Agitation Behavior Mapping Instrument. International Psychogeriatrics, 8(S3), 309-315. https://doi.org/10.1017/ S1041610297003530

Cohen-Mansfield, J. (2008). Agitated behavior in persons with dementia: The relationship between 
type of behavior, its frequency, and its disruptiveness. Journal of Psychiatric Research, 43(1), 64-69. https://doi.org/10.1016/j.jpsychires.2008.02.003

Cohen-Mansfield, J., \& Libin, A. (2005). Verbal and physical non-aggressive agitated behaviors in elderly persons with dementia: robustness of syndromes. Journal of Psychiatric Research, 39(3), 325-332. https://doi.org/10.1016/j.jpsychires.2004.08.009

Comité national d'éthique sur le vieillissement. (2015). Avis $\mathrm{n}^{\circ} 1$ aspects éthiques de l'utilisation de caméras vidéo dans les milieux de vie des aînés (p. 106). Québec, QC.

Contandriopoulos, A.-P., Champagne, F., Potvin, L., Denis, J., \& Boyle, P. (2005). Savoir préparer une recherche: la définir, la structurer, la financer. Montréal: gaëtan morin éditeur.

Dupuis, S. L., Wiersma, E., \& Loiselle, L. (2012). Pathologizing behavior: Meanings of behaviors in dementia care. Journal of Aging Studies, 26(2), 162-173. https://doi.org/10.1016/j.jaging.2011.12.001

Dyer, S. M., Harrison, S. L., Laver, K., Whitehead, C., \& Crotty, M. (2018). An overview of systematic reviews of pharmacological and non-pharmacological interventions for the treatment of behavioral and psychological symptoms of dementia. International Psychogeriatrics, 30(3), 295-309. https://doi. org/10.1017/S1041610217002344

Ferrero-arias, J., Goñi-imízcoz, M., González-bernal, J., Lara-ortega, F., Silva-gonzález, Á. da, \& Díez-lopez, M. (2011). The Efficacy of Nonpharmacological Treatment for Dementia-related Apathy. Alzheimer Disease \& Associated Disorders, 25(3), 213-219. https://doi.org/10.1097/ WAD.0b013e3182087dbc

Feteanu, D., Lopez-Tourres, F., \& Trivalle, C. (2005). La personne démente en institution. Psychologie and NeuroPsychiatrie Du Vieillissement, 3(1), 26-34.

Fraker, J., Kales, H. C., Blazek, M., Kavanagh, J., \& Gitlin, L. N. (2014). The Role of the Occupational Therapist in the Management of Neuropsychiatric Symptoms of Dementia in Clinical Settings. Occupational Therapy in Health Care, 28(1), 4-20. https://doi.org/10.3109/07380577.2013.867468

Hersch, E. (2008). Management of the behavioral and psychological symptoms of dementia. Clinical Interventions in Aging, Volume 2, 611-621. https:// doi.org/10.2147/CIA.S1698

Holden, R. J., \& Karsh, B.-T. (2010). The Technology Acceptance Model: Its past and its future in health care. Journal of Biomedical Informatics, 43(1), 159172. https://doi.org/10.1016/j.jbi.2009.07.002

Kales, H. C., Gitlin, L. N., \& Lyketsos, C. G. (2015). Assessment and management of behavioral and psychological symptoms of dementia. BMJ, 350, h369. https://doi.org/10.1136/bmj.h369

Lapierre, N., Carpentier, I., St-Arnaud, A., Ducharme, F., Meunier, J., Jobidon, M., \& Rousseau, J. (2016). Vidéosurveillance intelligente et détection des chutes : perception des professionnels et des gestionnaires: Intelligent videosurveillance and falls detection: Perceptions of professionals and managers. Canadian Journal of Occupational Therapy, 83(1), 33-41. https://doi.org/10.1177/0008417415580431

Lapierre, N., Meunier, J., St-Arnaud, A., Filiatrault, J.,
Paquin, M.-H., Duclos, C., Rousseau, J. (2018). Older women's perceptions of a programmable video monitoring system at home: A pilot study. Gerontechnology, 17(4).

Lapierre, N., Meunier, J., St-Arnaud, A., \& Rousseau, J. (2018). An intelligent video-monitoring system to detect falls: a proof of concept. Journal of Enabling Technologies, 12(4), 155-168. doi: 10.1108/JET-042018-0022

Londei, S., Rousseau, J., Ducharme, F., St-Arnaud, A., Meunier, J., Saint-Arnaud, J., \& Giroux, F. (2009). An intelligent videomonitoring system for fall detection at home: perceptions of elderly people. Journal of Telemedicine \& Telecare, 15(8), 383390. https://doi.org/10.1258/jtt.2009.090107

Lorusso, L. N., \& Bosch, S. J. (2018). Impact of Multisensory Environments on Behavior for People With Dementia: A Systematic Literature Review. The Gerontologist, 58(3), e168-e179. https://doi. org/10.1093/geront/gnw168

Macaulay, S. (2018). The Broken Lens of BPSD: Why We Need to Rethink the Way We Label the Behavior of People Who Live With Alzheimer Disease. Journal of the American Medical Directors Association, 19(2), 177-180. https://doi.org/10.1016/j. jamda.2017.11.009

McCraken, P., Kagan, L., \& Parmar, J. (2009). Reconnaître et traiter les symptômes comportementaux et psychologiques de la démence. La Revue Canadienne de La Maladie d'Alzheimer et Autres Démences, 12(1), 4-7.

Möhler, R., Renom, A., Renom, H., \& Meyer, G. (2018). Personally tailored activities for improving psychosocial outcomes for people with dementia in long-term care. Cochrane Database of Systematic Reviews, (2). https://doi.org/10.1002/14651858. CD009812.pub2

Möller, U. O., Kristensson, J., Midlöv, P., Ekdahl, C., \& Jakobsson, U. (2014). Effects of a One-Year HomeBased Case Management Intervention on Falls in Older People: A Randomized Controlled Trial. Journal of Aging and Physical Activity, 22(4), 457464 8p. https://doi.org/10.1123/JAPA.2013-0101

National Institute on Aging. (2016). World's older population grows dramatically. Retrieved from https:// www.nih.gov/news-events/news-releases/worldsolder-population-grows-dramatically

Neubauer, N. A., Lapierre, N., Ríos-Rincón, A., MiguelCruz, A., Rousseau, J., \& Liu, L. (2018). What do we know about technologies for dementia-related wandering? A scoping review, Examen de la portée: Que savons-nous à propos des technologies de gestion de l'errance liée à la démence?, Canadian Journal of Occupational Therapy, 85(3), 196208. https://doi.org/10.1177/0008417418777530

Niemeijer, A. R., Frederiks, B. J. M., Riphagen, I. I., Legemaate, J., Eefsting, J. A., \& Hertogh, C. M. P. M. (2010). Ethical and practical concerns of surveillance technologies in residential care for people with dementia or intellectual disabilities: an overview of the literature. International Psychogeriatrics, 22(7), 1129-1142. https://doi.org/10.1017/ S1041610210000037

Politis, A. M., Vozzella, S., Mayer, L. S., Onyike, C. 
U., Baker, A. S., \& Lyketsos, C. G. (2004). A randomized, controlled, clinical trial of activity therapy for apathy in patients with dementia residing in long-term care. International Journal of Geriatric Psychiatry, 19(11), 1087-1094. https://doi. org/10.1002/gps.1215

Qassem, T., Tadros, G., Moore, P., \& Xhafa, F. (2014). Emerging Technologies for Monitoring Behavioural and Psychological Symptoms of Dementia. 2014 Ninth International Conference on P2P, Parallel, Grid, Cloud and Internet Computing, 308-315. https://doi.org/10.1109/3PGCIC.2014.82

Rougier, C., St-Arnaud, A., Rousseau, J., \& Meunier, J. (2011). Video Surveillance for Fall Detection. In W. Lin (Ed.), InTech. Prof. Weiyao Lin.

Rousseau, J. (2016). L'Évaluation à domicile de I'interaction personne-environnement (ÉDIPE). In É. Trouvé, Agir sur l'environnement pour permettre les activités (pp. 475-489). Retrieved from https://www. calameo.com/read/000015856fd2d08ed51ad

Rousseau, J. (2017). Rousseau, J. (2017). Modèles généraux en ergothérapie: Le Modèle de compétence. In M. C. Morel-Bracq, Les modèles conceptuels en ergothérapie-Introduction aux concepts fondamentaux (2nd ed., pp. 107-119). Paris, France: De Boeck Supérieur.

Rousseau, J., Meunier, J., \& Saint-Arnaud, A. (2011). Les systèmes de détection des chutes à domicile : des outils pour donner l'alerte. Protégez-Vous, édition hors série(Guide pratique-Aide aux aînés : Tout ce que vous devez savoir pour accompagner les personnes âgées), 20-21.

Rousseau, J., Potvin, L., Dutil, E., \& Falta, P. (2002). Model of Competence: A Conceptual Framework for Understanding the Person-Environment Interaction for Persons with Motor Disabilities. Occupational Therapy in Health Care, 16(1), 15-36. https://doi.org/10.1080/J003v16n01_02

Rousseau, J., Potvin, L., Dutil, E., \& Falta, P. (2013). Home Assessment of Person-Environment Interaction (HoPE): Content Validation Process. 27(4). doi: https://doi.org/10.3109/07380577.2013.843114

Scales, K., Zimmerman, S., \& Miller, S. J. (2018). Evidence-Based Nonpharmacological Practices to Address Behavioral and Psychological Symptoms of Dementia. The Gerontologist, 58(suppl_1), S88S102. https://doi.org/10.1093/geront/gnx167

Treusch, Y., Majic, T., Page, J., Gutzmann, H., Heinz, A., \& Rapp, M. A. (2015). Apathy in nursing home residents with dementia: Results from a cluster-randomized controlled trial. European Psychiatry, 30(2), 251-257. https://doi.org/10.1016/j.eurpsy.2014.02.004

Vahia, I. V., Kamat, R., Vang, C., Posada, C., Ross, L., Oreck, S., Sewell, D. D. (2017). Use of Tablet Devices in the Management of Agitation Among Inpatients with Dementia: An Open-Label Study. The American Journal of Geriatric Psychiatry: Official Journal of the American Association for Geriatric Psychiatry, 25(8), 860-864. https://doi. org/10.1016/j.jagp.2016.07.011

World Health Organization. (2015). World report on ageing and health (p. 246). Geneva: World Health Organization.

Zaudig, M. (2000). A Risk-Benefit Assessment of Risperidone for the Treatment of Behavioural and Psychological Symptoms in Dementia. Drug Safety, 23(3), 183-195. https://doi.org/10.2165/00002018200023030-00002 